\title{
Identification of nontuberculous mycobacteria using commercial DNA probes and gene sequencing
}

Antonella Grottola, Massimino Messinò, Mariagrazia Apice, Alessia Di Nauta, Giuliana Fabio, Sara Tagliazucchi,
William Gennari, Anna Maria Teresa Sabbatini, Fabio Rumpianesi, Anna Fabio, Monica Pecorari
Dipartimento ad Attività Integrata dei Laboratori, Anatomia Patologica e Medicina Legale, Sezione di Microbiologia e Virologia, Azienda OspedalieroUniversitaria, Modena

Key words: Nontuberculous mycobacteria, GenoType Mycobacteria CM/AS, I6S rDNA, hsp65, sequencing

Identificazione di micobatteri non-tubercolari tramite DNA strip e sequenziamento genico

\section{SUMMARY}

Diagnosis of NonTuberculous Mycobacteria (NMT) infection frequently runs into difficulties regarding a precise definition of the strains. The use of molecular assays is the technique of choice for the identification of species, but commercial methods recognize only a limited number of species.

Aim of this study was molecular identification of NonTuberculous Mycobacteria (NTM) in clinical specimens using commercial methods and automated sequencing.

We analyzed 6192 clinical specimens for the isolation of Mycobacteria. One hundred and twelve strains of NTM were previously analyzed with GenoType Mycobacteria CM/AS kit and then with entire 16S rDNA and partial hsp65 sequencing.

100/I I2 NMT strains were identified with GenoType Mycobacteria CM/AS kit as: M. gordonae $\left(n^{\circ} 25\right), M . x e n o p i\left(n^{\circ} 24\right), M$. fortuitum $\left(n^{\circ} 15\right)$, M. avium ( $\left.n^{\circ} \mid 2\right), M$. intracellulare $\left(n^{\circ} 7\right), M$. chelonae $\left(n^{\circ} 6\right)$, M. malmoense $\left(n^{\circ} 4\right), M$. peregrinum $\left(n^{\circ} 3\right), M$. mucogenicum $\left(n^{\circ} I\right), M . k a n s a s i i\left(n^{\circ} I\right), M$. abscessus $\left(n^{\circ} \mathrm{I}\right), M$. heckeshornense $\left(n^{\circ} \mathrm{I}\right)$. Twelve unidentified strains were subjected to the entire I6S rDNA gene sequencing and nine were identified as M. arupense $\left(n^{\circ} 7\right)$, M. avium complex $\left(n^{\circ} \mathrm{I}\right)$, M. kumamotonense $\left(n^{\circ} \mathrm{I}\right)$. Three unidentified strains were subjected to partial hsp65 gene sequencing and one was identified as $M$. arupense.

Conclusions. Direct sequencing of entire 16SrDNA gene and of partial hsp65 gene appears to be a useful tool for the study of strains that are not identifiable by commercial methods. This new approach, applied to clinical diagnostic, allows also recognition of unusual strains or new species.

\section{INTRODUZIONE}

L'attuale interesse, sia clinico che epidemiologico, per le patologie sostenute dai Micobatteri Non Tubercolari (MNT) è notevolmente accresciuto e questo corrisponde a richieste diagnostiche sempre maggiori. La diagnosi di infezione da MNT, basata sui metodi classici di isolamento colturale, viene generalmente affiancata da test molecolari del commercio il cui limite è quello di identificare solo alcune fra le più frequenti specie isolate. Nella nostra esperienza, al fine di riconoscere ceppi di MNT non identificabili con i saggi del commercio, siamo ricorsi all'analisi di sequenza genica. Il target usato più di frequentemente per l'identificazione a livello di specie dei micobatteri, è il gene $16 \mathrm{~S}$ rDNA che codifica per l'RNA della subunità ribosomale $16 \mathrm{~S}(5,6,14,15)$. L'analisi di sequenza del gene $16 \mathrm{~S}$ si basa sul sequenziamento dell'intero gene, o in alternativa delle prime 540 basi, all'interno delle quali sono presenti 2 regioni ipervariabili A e $\mathrm{B}(3,11)$. Il sequenziamento dell'intero gene $16 \mathrm{~S}$ può consentire di identificare nuove specie e di permettere di differenziare tra loro specie strettamente correlate $(11,12)$.

L'utilizzo a scopi identificativi di un target genico unico può presentare dei limiti per cui, in talune situazioni, è necessario ricorrere all'utilizzo di target genici alternativi da affiancare all'analisi del 16SrDNA (7). I geni maggiormente studiati per l'identificazione dei micobatteri sono: hsp65, Internal Transcribed Spacer (ITS), rpoB, gyrB. In particolare l'hsp65 si è rivelato utile per l'identificazione di micobatteri a crescita lenta, l'rpoB per quelli a crescita rapida e l'ITS per l'identificazione di specie dei micobatteri appartenenti al M. avium complex (16).

Nel nostro studio 112 ceppi di micobatteri non tubercolari, isolati con le metodiche tradizionali, sono stati analizzati mediante il test molecolare Genotype Mycobacteria CM/AS (Hain, Lifescience, Nehren, Germany). I ceppi non identificati con il metodo commerciale sono stati sottoposti in prima battuta al sequenziamento dell'intero gene 16SrDNA e nei casi di mancata identificazione si è ricorso al sequenziamento di un frammento di $441 \mathrm{bp}$ del gene hsp65, codificante per una heat shock protein di $65 \mathrm{KDa}$.

Nel biennio 2007-2008, presso la Struttura Complessa di Microbiologia e Virologia del Policlinico di Modena, sono stati analizzati per la ricerca di micobatteri 6192 campioni clinici, provenienti da Modena e provincia. Da questi campioni sono stati isolati 112 ceppi di micobatteri atipici (MNT) provenienti da 110 pazienti. Dei 112 campioni clinici, 7/112 campioni erano positivi all'esame microscopico per la ricerca di batteri alcool-acido resistenti e 105/112 campioni erano negativi all'esame microscopico; di questi, 36 (15 lavaggi bronco alveolari, 16 espettorati, 2 essudati bronchiali, 1 urine, 1 liquido pleurico, 1 ascesso cerebrale) sono stati testati con il saggio Amplified Mycobacterium tuberculosis direct test (GenProbe - bioMérieux SA, Marcy l'Etoile, France) su richiesta clinica, risultando tutti negativi. Tutti i 112 ceppi isolati sono stati sottoposti allo stesso saggio con esito negativo. I ceppi di MNT provenivano per il $71 \%$ da pazienti di sesso maschile (78/110) e per il $29 \%$ da pazienti di sesso femminile $(32 / 110)$, con una netta prevalenza di isolamenti da materiali respiratori (bronco-aspirati e lavaggi bronco-alveolari: 90/112) rispetto a materiali provenienti da altri distretti (urine: 4/112; feci: 3/112; ascessi: 2/112; midollo osseo: $1 / 112$, sangue intero:1/112). Sul totale di 110 pazienti 24 erano immunodepressi; 6 pazienti erano positivi per il test dell'HIV e 2 pazienti presentavano una coinfezione con il virus HCV. Su questi 112 ceppi di MNT si è proceduto alla genotipizzazione previa estrazione dell'acido nucleico da coltura.

\section{Genotipizzazione}

Ibridazione Inversa

I ceppi di MNT sono stati analizzati con un metodo molecolare di genotipizzazione per l'identificazione di specie, previa estrazione degli acidi nucleici totali secondo le indicazioni del kit Genotype Mycobacterium CM/AS (10).

Sequenziamento

La reazione di amplificazione è stata realizzata in un volume finale di 50ul contenente $100 \mathrm{ng}$ di DNA estratto, $25 \mathrm{ul} \mathrm{di}$ Dream Taq Green PCR Master Mix (Fermentas International

\section{Corresponding author: Antonella Grottola}

Struttura Complessa di Microbiologia e Virologia, Azienda Integrata Ospedaliero-Universitaria, Policlinico,

Via del Pozzo 7I, 4II 00 Modena - Tel.: 059-4223752 - Fax: 059-4223625

E-mail: antonella.grottola@unimore.it 
Inc, Canada), 40 pmoli di ciascun primer. I primers usati per l'amplificazione dell'intero gene 16S erano 8FLP (AGAGTT TGATCATGGCTC) e 1492-Rv (GGTTACCTTGTTACGACTT) (9) che generano un frammento di 1503 bp (3' a $94^{\circ} \mathrm{C}$; 40 cicli a $94^{\circ} \mathrm{C}-30^{\prime}$ ", $55^{\circ} \mathrm{C}-30$ ", $72^{\circ} \mathrm{C}$ per 1 ' 40 "; estensione finale a $\left.72^{\circ} \mathrm{C}-10^{\prime}\right)$.

I primers usati per l'amplificazione parziale del gene hsp65 erano: Hsp65 Fw (ACCAACGATGGTGTGTCCAT) e Hsp65 Rv (CTTGTCGAACCGCATACCCT) (13) che generano un frammento di 441 bp utilizzando il seguente programma: $3^{\prime}$ a $94^{\circ} \mathrm{C}$; 35 cicli a $94^{\circ} \mathrm{C}-30$ ", $56^{\circ} \mathrm{C}-30^{\prime}, 72^{\circ} \mathrm{C}$ per $30^{\prime \prime}$; estensione finale a $72^{\circ} \mathrm{C}-10^{\prime}$. Il prodotto di amplificazione era controllato su gel d'agarosio all' $1.5 \%$, purificato mediante kit commerciale (QiaQuick PCR purification kit, Qiagen, Milano, Italia) e sottoposto a reazione di sequenziamento bidirezionale usando il Big-dye terminator Kit v.1 (Applied Biosystem, Foster City, CA). Il prodotto di sequenziamento veniva analizzato tramite elettroforesi capillare su sequenziatore automatizzato a fluorescenza (ABI PRISM 3100). Le sequenze ottenute sono state analizzate con un software di allineamento (SeqScape, Applied Biosystem), ottenendo una sequenza di consenso. Tale sequenza è stata inserita in GeneBank Database dell'NIH (2) per il confronto, tramite il software BLAST, con le altre sequenze depositate al fine di ottenere l'identificazione di specie. A tale scopo veniva considerata significativa una percentuale di omologia rilevata rispetto ad un reference strain pari al $100 \%$ oppure al $99 \%$ con una tolleranza di mismatch dello $0,2 \%$ per il gene $16 \mathrm{~S}$ o dell' $1 \%$ per il gene hsp65.

I 112 ceppi di MNT, provenienti dai 6192 campioni clinici sottoposti alla ricerca di micobatteri, sono stati isolati da materiali clinici di varia natura, come descritto in Tabella 1. Cento dei 112 ceppi di MNT (89\%) sono stati identificati con i kit del commercio Genotype Mycobacterium CM/AS come appartenenti alle specie riportate in Tabella 2. In particolare il kit Genotype Mycobacterium CM/AS ha permesso di identificare: M. gordonae ( $\mathrm{n}^{\circ} 25$ ceppi), M. xenopi ( $\mathrm{n}^{\circ} 24$ ceppi), M. fortuitum1/2 ( $\mathrm{n}^{\circ} 15$ ceppi), M. avium ( $\mathrm{n}^{\circ} 12$ ceppi), M. intracellulare ( ${ }^{\circ} 7$ ceppi), M. chelonae ( $\mathrm{n}^{\circ} 6$ ceppi), M. malmoense ( $\mathrm{n}^{\circ} 4$ ceppi), M. peregrinum ( $\mathrm{n}^{\circ} 3$ ceppi), M. тисоgenicum ( $\mathrm{n}^{\circ} 1$ ceppo), M. kansasii ( $\mathrm{n}^{\circ} 1$ ceppo), M. abscessus ( $\mathrm{n}^{\circ} 1$ ceppo), M. heckeshornense ( $\mathrm{n}^{\circ} 1$ ceppo).

Per quanto riguarda la corrispondenza ai criteri microbiologici di diagnosi delle patologie polmonari da MNT, in 29/110 pazienti i ceppi di MNT erano stati isolati da almeno un campione di BAL e in 8/110 si era avuto un isolamento di MNT da essudato bronchiale; tutto ciò depone per una loro significatività clinica rispondente ai criteri dell'American Thoracic Society (ATS) (1). In particolare i ceppi isolati da BAL erano M. gordonae ( $\mathrm{n}^{\circ} 8$ ceppi), M. xenopi ( ${ }^{\circ} 6$ ceppi), M. avium ( $\mathrm{n}^{\circ} 4$ ceppi), M. intracellulare ( ${ }^{\circ} 4$ ceppi), M. malmoense $\left(\mathrm{n}^{\circ} 2\right.$ ceppi), M. abscessus ( $\mathrm{n}^{\circ} 1$ ceppo), M. arupense $\left(\mathrm{n}^{\circ} 1\right.$ ceppo), M. fortuitum $1 / 2$ ( $\mathrm{n}^{\circ} 1$ ceppo), M. peregrinum $\left(\mathrm{n}^{\circ} 1\right.$ ceppo), M. heckeshornense ( ${ }^{\circ} 1$ ceppo). Di questi 29 pazienti, 5 erano immunodepressi. In 5 pazienti erano state ottenute colture positive da almeno due differenti campioni di espettorato. I ceppi isolati erano stati identificati come $M$. malmoense (1), M. peregrinum (1), M. xenopi (3). Dei 5 pazienti uno era immunodepresso.

I 12 ceppi non identificati sono stati sottoposti al sequenziamento del gene $16 \mathrm{~S}$ rDNA; di questi 9 ceppi sono stati riconosciuti come $M$. arupense (7), M. avium complex (1) e $M$. kumamotonense (1). L'analisi del gene $16 \mathrm{~S}$ rDNA non ha permesso di identificare i restanti 3 ceppi che sono stati, perciò, sottoposti al sequenziamento del gene hsp65.
Grazie a quest'analisi un ceppo è risultato appartenere alla specie M. arupense. Gli altri 2 ceppi non sono stati identificati con nessuno dei metodi di analisi utilizzati.

Anche per i 10 ceppi identificati con l'analisi di sequenza è stata valutata la significatività clinica. In particolare, 3 ceppi di $M$. arupense sono risultati significativi da un punto di vista clinico in quanto per essi sono state ottenute colture positive a partire da due separati campioni di espettorato, come dai criteri di valutazione dettati dall'ATS. Per 4 ceppi di cui 3 di M. arupense e 1 ceppo di $M$. kumamotonense, era stata effettuata un'unica coltura su espettorato, risultata positiva, mancando, in questi casi, la significatività clinica. I restanti 3 ceppi sono stati isolati da campioni ad elevata significatività clinica quali essudato bronchiale (1 ceppo di $M$. arupense e 1 ceppo di $M$. avium complex) e BAL (1 ceppo di $M$. arupense).

Dei pazienti dai quali sono stati isolati i 10 ceppi identificati con l'analisi di sequenza, un paziente era immunodepresso ed un altro era risultato positivo al test dell'HIV; da entrambi erano stati isolati ceppi di M. arupense.

L'approccio alle patologie sostenute dai Micobatteri Non Tubercolari (MNT) incontra frequentemente difficoltà sul piano diagnostico riguardo ad una precisa definizione dei ceppi. L'impiego di saggi molecolari rappresenta la tecnica elettiva per l'identificazione di specie anche se, i metodi commerciali attualmente disponibili basati sulle sonde di DNA, presentano dei limiti perchè riconoscono esclusivamente le specie più spesso isolate. I metodi molecolari del commercio, per quanto sensibili e specifici, non sono in grado di tipizzare tutte le specie di MNT descritte; in particolare il GenoType Mycobacteria CM/AS permette di identificare solo 32 delle specie di MNT più frequentemente isolate nei campioni clinici. Per questo motivo nasce l'esigenza di identificare tutte le specie di ceppi isolabili e a tale scopo è necessario ricorrere a metodi alternativi che siano altamente specifici. In questo studio si è ricorsi all'analisi di sequenza di due target genici, il gene 16SrDNA ed il gene hsp65, correntemente utilizzati nelle analisi tassonomiche e filogenetiche (5), ma indiscutibilmente utili anche dal punto di vista diagnostico.

Nella nostra esperienza l'analisi di sequenza dei geni 16SrDNA e hsp65 ha permesso di giungere all'attribuzione di specie per la maggior parte dei ceppi non identificati con il metodo commerciale (10/12 ceppi). Solo per due ceppi non è stata raggiunta una precisa definizione di specie. In particolare è stato possibile identificare un ceppo di M. kumamotonense e otto ceppi di $M$. arupense: questo rappresenta un dato di estremo interesse poiché si tratta di specie di recente classificazione $(8,4)$ di cui non è stata ancora ampiamente indagata l'importanza clinica. A tale proposito è importante sottolineare che cinque degli otto ceppi di M. arupense da noi isolati mostravano avere significatività clinica in aderenza ai criteri di valutazione microbiologici dettati dall' ATS. Per il $M$. kumamotonense non era possibile fare la stessa valutazione in quanto era stata effettuata un'unica coltura su espetto-

Tabella I. Specie di MNT identificate dai materiali clinici di isolamento

\begin{tabular}{|c|c|}
\hline Materiale clinico & Specie identificate \\
\hline \multirow[t]{3}{*}{$\mathrm{BAL}$} & M. abscessus (I), M. arupense (I), M. avium (4), \\
\hline & M. fortuitum I/2 (I), M. gordonae (8), M. intracellulare (4), \\
\hline & M. malmoense (2), M. peregrinum (I), M. xenopi (6), M. heckeshornense (I) \\
\hline \multirow[t]{4}{*}{ Espettorato } & M. arupense (6), M. avium (4), M. chelonae (4), \\
\hline & M. fortuitum I/2 (I2), M. gordonae (I3), M. intracellulare (2), \\
\hline & M. kansasii (I), M. kumamotonense (I), M. malmoense (2), \\
\hline & M. peregrinum (2), M. xenopi (13), \\
\hline \multirow[t]{2}{*}{ Essudato bronchiale } & M. arupense (I), M. chelonae (2), M. intracellulare (I), \\
\hline & M. mucogenicum (I), M. xenopi (2), M. avium (I) \\
\hline Liquido pleurico & M. gordonae (2) \\
\hline Urine & M. fortuitum I/2 (2), M. gordonae (I), M. xenopi (I) \\
\hline Feci & M. gordonae (I), M. xenopi (2) \\
\hline Ascesso & M. avium (2) \\
\hline Midollo osseo & M. avium (I) \\
\hline Sangue & M. avium (I) \\
\hline
\end{tabular}


rato, risultata positiva.

Il $M$. kumamotonenese, è stato isolato per la prima volta in Giappone nel 2001 da una paziente con lesioni polmonari visibili radiograficamente, mentre il $M$. arupense è stato isolato per la prima volta negli USA nel 2001, da una biopsia tendinea. I dati fenotipici insieme a quelli filogenetici collocano $M$. kumamotonense e $M$. arupense nel $M$. terrae complex $(8,4)$.
Da questi risultati emerge che il sequenziamento diretto del gene $16 \mathrm{~S}$ rDNA si dimostra il metodo più adatto e completo per una corretta tipizzazione, anche se in alcuni casi non è sufficientemente discriminante per l'identificazione di tutti i micobatteri non tubercolari. Per ovviare a questo limite abbiamo introdotto l'analisi di sequenza per il gene hsp 65 e per l'immediato futuro intendiamo estenderla anche ai target genici rpoB e ITS.

Tabella 2. Risultati della genotipizzazione di I I 2 ceppi di MNT ottenuti mediante Genotype Mycobacterium CM/AS e sequenziamento genico

\begin{tabular}{|c|c|c|c|}
\hline Specie identificate & $\begin{array}{l}\text { Numero di ceppi identificati con } \\
\text { Genotype Mycobacterium CM/AS }\end{array}$ & $\begin{array}{c}\text { Numero di ceppi identificati } \\
\text { con sequenziamento completo } \\
\text { del gene I6SrDNA }\end{array}$ & $\begin{array}{l}\text { Numero di ceppi identificati } \\
\text { con sequenziamento parziale } \\
\text { del gene hsp } 65\end{array}$ \\
\hline M. gordonae & 25 & - & 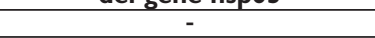 \\
\hline M. xenopi & 24 & - & - \\
\hline M. fortuitum I/2 & 15 & - & - \\
\hline M. avium & 12 & I & - \\
\hline M. intracellulare & 7 & - & - \\
\hline M. chelonae & 6 & - & - \\
\hline M. malmoense & 4 & - & - \\
\hline M. peregrinum & 3 & - & - \\
\hline M. mucogenicum & I & - & - \\
\hline M. kansasii & I & - & - \\
\hline M. abscessus & $\mathrm{I}$ & - & - \\
\hline M. heckeshornense & $\mathrm{I}$ & - & - \\
\hline M. arupense & - & 7 & $\mathrm{I}$ \\
\hline M. kumamotonense & - & 1 & - \\
\hline Totale ceppi identificati & $100 / 112$ & $9 / 12$ & $1 / 3$ \\
\hline Totale ceppi non identifi & $12 /|| 2$ & $3 / 12$ & $2 / 3$ \\
\hline
\end{tabular}

\section{BIBLIOGRAFIA}

1. American Thoracic Society. 1987. Mycobacterioses and the acquired immunodeficiency syndrome. Joint Position Paper of the American Thoracic Society and the Centers for Disease Control. Am Rev Respir Dis 136:492-6.

2. Benson DA, Karsch-Mizrachi I, Lipman DJ, Ostell J, Wheeler DL. GenBank. Nucleic Acids Research, 2008, Jan;36(Database issue):D25-30)

3. Bottger EC. 1996. Approaches for identification of microorganisms. ASM News 62:247-250

4. Cloud JL, Meyer JJ, Pounder JI, Jost Jr KC, Sweeney A, Carroll KC And Woods GL. 2006. Mycobacterium arupense sp. nov., a non-chromogenic bacterium isolated from clinical specimens. Int. J. Syst. Evol. Microbiol., , $56,1413-1418$

5. Drancourt M, Bollet C, Carlioz A, Martelin R, Gayral JP, Raoult D. 2000 $16 \mathrm{~S}$ ribosomal DNA sequence analysis of a large collection of environmental and clinical unidentifiable bacterial isolates. J Clin Microbiol 38:3623-30.

6. Harmsen D, Karch H. 2004. 16s rRNA for diagnosis pathogens: a living tree. ASM News 70:p. 19-24.

7. Kim H, Kim SH, Shim TS, Kim MN, Bai GH, Park YG, Lee SH, Chae GT, Cha CY, Kook YH, Kim BJ. 2005. Differentiation of Mycobacterium species by analysis of the heat-shock protein 65 gene (hsp65). Int J Syst Evol Microbiol. Jul;55(Pt 4):1649-56.

8. Masaki T, Ohkusu K, Hata H, Fujiwara N, Iihara H, Yamada-Noda M, Nhung PH, Hayashi M, Asano Y, Kawamura Y, Ezaki T. 2006. Mycobacterium kumamotonense Sp. Nov. recovered from clinical specimen and the first isolation report of Mycobacterium arupense in Japan:
Novel slowly growing, nonchromogenic clinical isolates related to Mycobacterium terrae complex. Microbiol Immunol 50:889-97.

9. Relman DA, Loutit JS, Schmidt TM, Falkow S, Tompkins LS. The agent of bacillary angiomatosis. An approach to the identification of uncultured pathogens. N Engl J Med. 1990, Dec 6;323(23):1573-80.

10. Richter E, Rusch-Gerdes S, Hillemann D. 2006. Evaluation of the GenoType Mycobacterium assay for identification of mycobacterial species from cultures. Antimicrob.Agents Chemother. 44:1769-1775.

11. Sacchi CT, Whitney AM, Mayer LW, Morey R, Steigerwalt A, Boras A Weyant RS, Popovic T. 2002. Sequencing of 16S rRNA gene: a rapid tool for identification of Bacillus anthracis. Emerg Infect Dis 8:1117-23.

12. Sacchi CT, Whitney AM, Reeves MW, Mayer LW, Popovic T. 2002. Sequence diversity of Neisseria meningitidis 16S rRNA genes and use of $16 \mathrm{~S}$ rRNA gene sequencing as a molecular subtyping tool. J Clin Microbiol 40:4520-7.

13. Telenti A, Marchesi F, Balz M, Bally F, Böttger EC, Bodmer T. Rapid identification of mycobacteria to the species level by polymerase chain reaction and restriction enzyme analysis. J Clin Microbiol. 1993, Feb;31(2):175-8.

14. Tortoli E. 2003. Impact of genotypic studies on mycobacterial taxonomy: the new mycobacteria of the 1990s. Clin Microbiol Rev 16:319-54.

15. Turenne CY, Tschetter L, Wolfe J, Kabani A. 2001. Necessity of qualitycontrolled 16S rRNA gene sequence databases: identifying nontuberculous Mycobacterium species. J Clin Microbiol 39:3637-48.

16. Woo PC, Lau SK, Teng JL, Tse H, Yuen KY. 2008. Then and now: use of $16 \mathrm{~S}$ rDNA gene sequencing for bacterial identification and discovery of novel bacteria in clinical microbiology laboratories. Clin Microbiol Infect. Oct;14(10):908-34. Review. 